\title{
Vivre le temps dans En vieillissant les hommes pleurent de Jean-Luc Seigle
}

\author{
Aliaa Ahmed Mohamed Abd El-Wahed \\ Professeur-adjoint \\ Faculté de pédagogie-Université de Canal El Suez
}


$-42-$

PDF created with pdfFactory Pro trial version www.pdffactory.com 


\section{Résumé}

\section{Vivre le temps dans En vieillissant les hommes pleurent de Jean-Luc Seigle}

Dans cette recherche, on aborde la conception de vivre le temps. Certains s'accrochent tellement au passé et se détachent du présent au point que ce dernier devient une menace. Ceci est dû à ce que le présent abolit le passé et renouvelle les conceptions d'autrefois. Ces personnes anticipent leur fin car ils refusent le présent qui précipite le temps et s'enferment loin du monde avec lequel ils n'arrivent jamais à s'adapter.

D'autres vivent le présent sans passé qui devient à son tour un ennemi. Cela tient du fait que le passé fait perdre le plaisir du présent par les mauvais souvenirs, et empêche toute nouveauté. Ils sont ainsi en changement continu. Cependant, ces personnes perdent leur identité car ils oublient leur racine et leur histoire. En plus, ils sont instables essayant de garder, en vain, le présent qui s'écoule et devient plus tard un passé.

$\mathrm{Ni}$ les uns ni les autres vivent joyeusement. En revanche, ils sont en lutte permanente contre un temps menaçant.

L'étude répond à la problématique suivante : Peut-on vivre le temps sans lutte malgré sa fuite et ses défis ? Le temps pourrait-il être source du bonheur ?

Alors, l'homme doit d'abord respecter la linéarité du temps. Il réconcilie avec lui en vivant les deux temps en harmonie. L'homme gère à sa façon le temps d'une manière objective. Le roman présente plusieurs moyens pour vivre les deux temps simultanément : 
D'abord, en sauvegardant les objets antiques qui témoignent des siècles lointains. L'homme revit deux époques en même temps.

De même, les œuvres littéraires d'auparavant transmettent un mode de vie, des attitudes sociales et humaines et des traditions qui guident l'homme du présent à comprendre et à interpréter ce qui se passe au quotidien.

Enfin, on peut renouer avec le passé et le présent en se référant à l'Histoire. On observe les épreuves des grandes personnalités historiques et leur pensée créatrice en faveur de leur pays et de leur société. On applique leurs idées au quotidien afin d'améliorer la vie.

Ces hommes bénéficient de vivre un temps harmonieux dont les conséquences sont les suivantes :

- L'homme n'est plus en lutte contre un temps ennemi et il vit en repos.

- Il maîtrise le temps et le gère objectivement.

- Il investit le temps en interprétant les mystères du présent à la lumière des épreuves des prédécesseurs.

- Vivre le présent avec le passé enrichit la culture de l'homme et lui permet plusieurs rencontres avec les anciens et les contemporains.

- Il n'est plus surpris des incidents du jour car il les a déjà sus à travers le passé puisque les événements se répètent au cours des siècles.

Finalement, l'auteur transmet le message suivant: au moment où l'homme respecte la succession du temps : du passé au présent et les investit, le temps devient une source de bonheur et de construction et renonce à être une source de malheur et de destruction. 
تثخص

معايشة الزمن في رواية الرجال يبكون عندما يكبرون لجون لوك سياجل يتناول البحث مفهوم معايشة الزمن ـ فهناك من يتمسكون بالعيش في الماضي دون الحاضر حتي اصبح الحاضر تمديدا لهم لانه يمحو ذكريات الماضي و يجري بالزمن و يغير من المفاهيم القديمة و

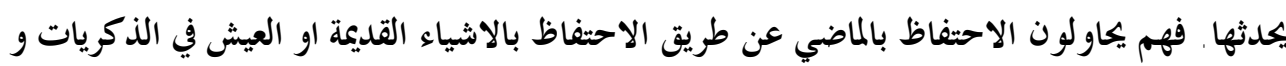

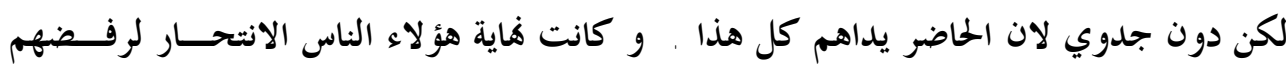
الحاضر الذي يداهمهم بسرعته و بسبب ايضا عدم التاقلم مع غيرهم و انطواءهم و البعد عن الحياة

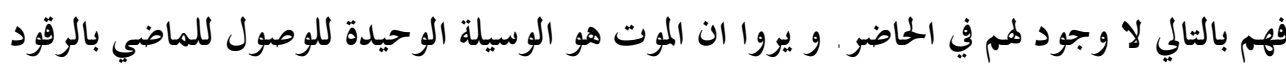
بجانب اجدادهم

وهناك من يعيشون الحاضر بلا ماضي , فينسوه تماما و ينظرون اليه كانه عدو هــــم لانسـهـ يفقدهم متع الحاضر بذكرياته الاليمة و حداثته بمفاهيمه القديمة . فيحافظوا علي الحاضر بتغيير كل من حولمم من قديم و يغيرون شر كاء حياتم و يتمسكون بالافكار الحديثة و يطمسون كل معسـالم

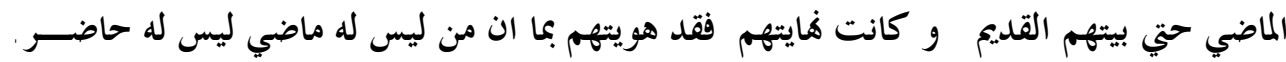

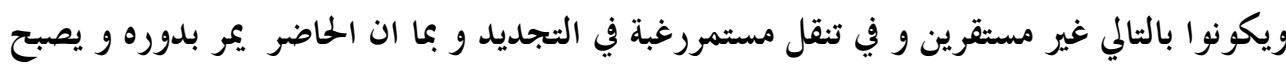
ماضي فهم يعيشون بلا رضا و لا راحة. كل هؤلاء لا يجدون سعادة و لا هدوء في العيش لافمم في صراع مستمر غـــــ الماضــي او الحاضر .

و الاشكالية التي يطرحها البحث هي: هل يستطيع الانسان ان يعيش الزمن دون صراع و

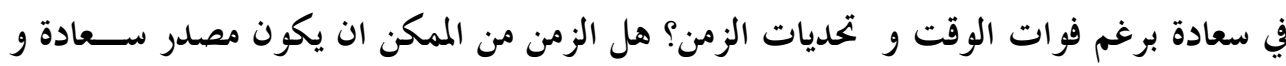
ليس شقاء؟

لكي ينعم الانسان بععايشة الزمن في سلام و راحة عليه باحترام تعاقب الزمن من ماضسـي و

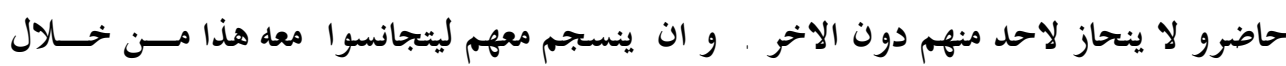
معايشة الماضي مع الحاضر في منظومة يتحكم فيها الانسان و يديرها بنفسه

$-45-$ 
هناك طرق متعددة لربط الماضي بالحاضر بالانسان من خلال الحفاظ علي المقتنيات القديمة التي تشهد الازمان السالفة و تذكر الانسان بما حدث في الماضي و ليتمتع باحساس انه يعيش حقبتين

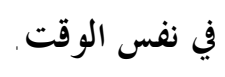

هناك ايضا الروايات التي تحكي طريقة حياة و سلو كيات و مواقف زمن ماضي. يصبح العمل

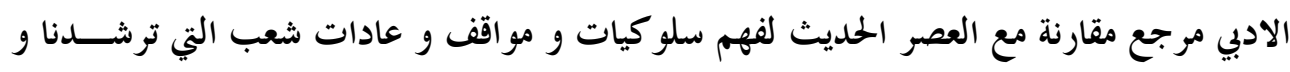
تفسر لنا ما يدور من احداث في الحاضر لا نفهمها لإنه و اخيرا هناك ربط بين الحاضر بالماضي عبر استعادة التاريخ وخبرات الشخصيات التاريخية الكبيرة و كيف كانوا يفكرون و يقيمون مشاريعهم و يخططون استطاع هؤلاء الناس معايشة زمنهم باستمتاع و باسلوب موضوعي و ليس نفسي و ذلك له

$$
\text { عظيم الاثر علي حياة الانسان في امور كثيرة و هي: }
$$

- عدم الشعور ابدا بالصراع مع الوقت لان الانسان مترابط مع الحاضر و و الماضي في انسجام المان

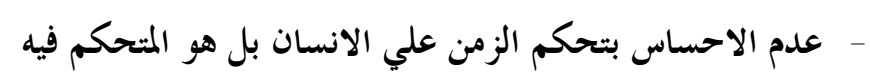

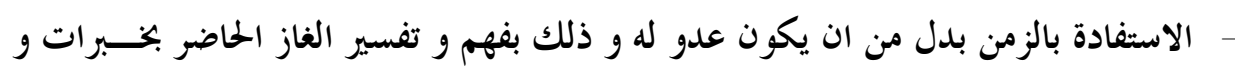
مواقف الماضي - معايشة الحاضر مع الماضي يزداد الانسان ثقافات متعددة و معرفة بشخصيات عديـــدة و لا لا ماضل

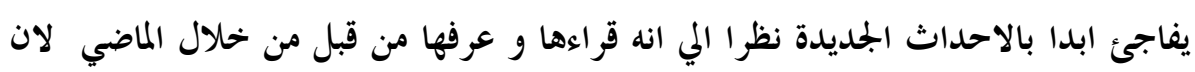

$$
\text { احداث الحياة تتكرر. }
$$

و ختاما اراد الكاتب توصيل هذة الرسالة لنا: طالما الانسان يحترم مفهوم تعاقب الزمن من

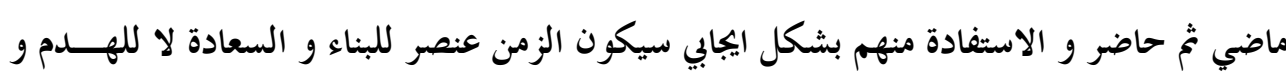
الشقاء.

$-46-$ 


\section{Introduction}

«Le romancier ne peut éviter de s'attaquer au temps, et la manière dont il le traite est un indice qui permet de juger à quel point il maîtrise son art. »1

À travers son roman, l'écrivain Jean-Luc Seigle aborde la question du temps : on y découvre sa perception du passé et du présent par le biais de ses personnages.

Certains aiment vivre dans le passé, et s'enferment dans leurs souvenirs, se réfugiant dans leur jeunesse et leur enfance. Ils nient alors complètement le présent, qui leur paraît menaçant car il abolit leur passé intime.

D'autres perçoivent le passé comme un ennemi qu'ils doivent combattre, et changent constamment tout ce qui les entoure. De cette façon, ils détruisent toute empreinte du passé afin de sauvegarder l'instant présent.

Par conséquent, les personnages sont en lutte permanente contre un temps ennemi, protègent leur temps favori, et sont en proie à la déception car le temps est irréversible et que son passage est inéluctable.

1 Edward-T Hall, La Dance de la Vie, Temps culturel, Temps Vécu, Seuil, Paris, 1992, p 157 
Pourtant, ils ne peuvent ni conserver le passé intime lié à l'écoulement du temps, ni empêcher le présent de ne pas être un passé. Leurs efforts n'entravent pas le pouvoir de l'instant qui s'inscrit avec eux dans un rapport aléatoire.

Roger Dadoun interprète la relation conflictuelle entre le temps et l'homme à ses mots:

"La violence du temps creuse dans l'âme des pertes irrémédiables -mémoire fissurée, croulante; de même elle creuse dans la chair, avec une aveuglante et précise efficacité, cette empreinte qui se nomme vieillissement" ${ }^{2}$

Ils sont alors en lutte permanente contre la linéarité du temps qui leur fait perdre soit le passé, soit le présent.

À cet égard, on se demande s'il est possible de jouir du temps et d'éviter de concevoir son infinie rétrogradation comme un processus menaçant. Autrement, peut-on vivre le temps sans lutte malgré sa fuite et ses défis ?

Basée sur plusieurs critiques et s'inscrivant dans une approche philosophique, notre étude vise à répondre à cette problématique en examinant trois manières proposées par le roman de vivre le temps :

- Le refus du présent ;

2 Roger Dadoun, «La violence», coll. Optiques- Philosophie, Hatier,1993, p. 53. 
- Le refus du passé ;

- La jouissance du temps.

\section{Le refus du présent}

Certains hommes choisissent de s'enfermer dans leur passé, suivant en cela ce qu'a souligné Heidegger dans son livre Etre et Temps : «je suis mon passé, mon passé dure en moi ». ${ }^{3}$

Selon Albert, le père de famille, qui refuse le présent, son travail est pénible, et vivre l'instant n'est qu'un enfer : «un temps durant lequel il s'était lui-même condamné aux travaux forcés $»^{4}$. Ce temps l'éloigne de son sujet favori : le passé.

Obsédé par les souvenirs, Albert n'aime que ce qui suscite l'odeur du temps vécu.

«Il n'aimait rien plus que cette odeur préhistorique quand il rentrait de l'usine le matin très tôt après une nuit dans l'enfer des pneus ${ }^{5}$

Pour lui, revivre le passé signifie retourner à ses origines campagnardes. Albert, paysan qui a été obligé de quitter sa terre et celle de sa famille pour gagner sa vie en travaillant à l'usine, trouve que le présent n'est qu'une suite de contraintes. Il n'en

3 Martin Heidegger (trad. François Vezin), Être et Temps, Paris, Gallimard, 1986, p589

4 Jean-Luc -Seigle, En vieillissant les hommes pleurent, Flammarion, 2012, p202

5 lbid. p 16 
retient que les terres. Celles-ci lui rappellent ses ancêtres qui les ont cultivées pendant des siècles.

«Albert n'avait pas de plus grande satisfaction que de redevenir un paysan et cela même si le travail de la terre rognait sur ses heures de sommeil $»^{6}$ afin de ressusciter les moments du temps passé et d'abolir le présent.

Il passe son temps à cultiver la terre de ses grands-parents, d'où «les images se soulevaient de la terre comme des mirages $d u$ passé $»^{7}$, et où les arbres évoquent la métaphore des racines qui, comme les souvenirs pour l'homme, sont inséparables de la plante.

Chaque fois qu'il travaille son jardin ou sa vigne, lui revient dans sa mémoire une image qui reste constante à travers le temps et les générations : celle du geste du semeur. L'ampleur du geste de son arrière-grand-père, de son grand-père et de son père jetant devant eux la semence dans la terre labourée réveille en lui la mémoire des labours de l'histoire.

Les champs ressuscitent le temps passé, et lui permettent de vivre dans les souvenirs qui, selon lui, constituent un défi du temps. Pour le héros de Seigle, les réminiscences sont

6 Ibid. p 19

7 Ibid. p 136 
concrétisées par les lieux des ancêtres, comme le champ de vigne. Son passé traduit son existence dans le monde comme le souligne Heidegger :

«Cet "être-été » donc est partie intégrante de l'existence du Dasein venant à soi, libre. $\rangle^{8}$; le personnage s'identifie au temps passé et refuse son identité du présent. Il affirme : «je n'aime pas qui je suis. Je n'aime pas ce qu'il faudrait que je sois, je n'aime pas me réjouir de cette vie-là ${ }^{9}$

Par conséquent, Albert vit dans un monde qui lui est propre, claustré dans sa mémoire, où il se connaît comme adolescent et jeune. Ses souvenirs refluent d'un passé lointain, alors que les paysages évoquent l'antériorité, et suggèrent un nouveau monde auquel il s'accroche, il s'exclame : «Je suis d'un autre temps ${ }^{10}$.

Cela se traduit également par son allure physique et vestimentaire. Albert refuse d'être un homme à l'apparence moderne : "C'était toujours une comédie quand il s'agissait de lui faire acheter une veste ou un pantalon ${ }^{11}$

Il préfère porter ses bleus, son ancien costume et sa canadienne dont il retire la doublure de peau de mouton en été.

8 Martin Heidegger (trad. François Vezin), Être et Temps,

Paris, Gallimard, 1986, p 536

9 Jean Luc Seigle, Op.cit. p 195

10 Idem.

11 lbid. p 42

$-51-$ 
Ces habits traduisent son image de lui-même, le renvoient à un autre monde, lui rappellent son allure d'antan et celle des gens qui lui sont chers. De cette façon, il se retire du présent.

Afin de revivre pleinement l'ancien temps, il s'obstine à se trouver dans le lieu qui évoque le passé. Sa place préférée est celle du vieux fauteuil, près de l'ancienne cheminée où a été installé un «poêle à mazout» désuet qui ne fonctionne plus mais qui atteste le passé.

Le héros se sent à l'intérieur du temps. Maurice MerleauPonty a expliqué que : l'homme ne se trouve pas seulement dans l'espace, mais qu'il habite l'espace ${ }^{12}$. Ainsi, Albert s'attache à cette place «que tous les vieux avaient occupée de tout temps $»^{13}$ Cet espace l'aide à remonter à la surface des réminiscences enfouies durant plusieurs années.

Enchaîné par ses souvenirs, Albert s'enferme dans un temps sclérosé comme David Hume l'a bien signalé qu"Un homme plongé dans un profond sommeil, ou fermement occupé par une seule pensée, ne sent pas le temps, et selon que ses perceptions se succèdent avec plus ou moins de rapidité, la même durée

12 Miklos Vetö, L'eidétique de l'espace chez Merleau-Ponty, revue Archive de philosophie, Université de Poitiers, article no3, 2008, en (https://www.cairn.info)

13 Ibid. p 75 
apparaît plus ou moins longue à son imagination [...]. ${ }^{14}$ le héros évite de cette façon tout engagement dans le présent. Il trouve dans la distraction une échappatoire du quotidien :

"Il avait toujours eu besoin de faire autre chose. Si ce n'était pas le jardin, c'était ses pendules, et si ce n'était pas ses réveils cassés, c'était l'accordéon, si ce n'était pas la musique, c'était la vigne qu'il fallait sarcler. ${ }^{15}$

Il devient l'héritier et le possesseur de son passé. Une série de souvenirs défile dans sa conscience où il se rappelle son enfance, son père, les ordres paternels, son entrée dans la chambre de ses parents.

De même, la mère d'Albert, Madeleine Chassaing, participe avec son fils à cet amour d'antan et vit avec lui dans les souvenirs. Elle sombre dans un passé douloureux, durant lequel elle a servi son mari malade et a cultivé ses champs.

Le couple mère-enfant vit dans un univers clos. Madeleine est perdue dans sa mémoire. Elle ne ressent plus ni le présent ni son corps, mais garde toujours le souvenir de la grande travailleuse qu'elle a été.

14 David Hume, Traité de la nature humaine, livre I et appendice, traduction de Philippe Baranger et Philippe Saltel, GF- Flammarion, Paris, 1995, p. 86.

15 Jean-Luc- Seigle, Op.cit. p. 126 
Emilie Charlet souligne à ce sujet que lorsque l'homme partage avec un autre ses souvenirs ; ce partenaire lui sert de “ support grâce auquel un ensemble donné de personnes se reconnaît comme partageant une même expérience du temps qui passe. ${ }^{16}$

En effet, Albert et sa mère s'encouragent à maintenir la moindre trace du passé. Ensemble, ils font une remontée fulgurante dans le temps, et se souviennent fréquemment de leur jeunesse.

Madeleine se rappelle les champs, son mari, et préfère se détacher du présent et s'asseoir dans le jardin, sous le cerisier, échappant ainsi à toute situation qui l'attache au quotidien.

Quant à Albert, il vit les souvenirs avec émotions et perturbations. Pourtant, il est indifférent face aux faits du quotidien :

«Toutes ses émotions revenaient en lui dans un désordre incontrôlable. Ce fut un frémissement sous sa peau d'homme qui, étrangement, le rafraîchit jusqu'à provoquer une multitude de décharges électriques qui excitaient son plaisir et le laissaient

16Emilie Charlet, L'Objet, la Relation., revue Agôn, dossiers no 4, 2011. En (http//agon.ens-lyon.fr/index.php? id=2152.) 
tout étonné. Albert, au cour du champ de son enfance, dans ce paysage millénaire, jouissait soudain d'être vivant ${ }^{17}$

D'ailleurs, le couple mère-fils renonce à se détacher de son accent patois et insiste pour l'utiliser afin de se rappeler le passé : «Il suffisait qu'il (Albert) se mette à lui (sa mère) parler dans sa langue ancienne pour qu'elle refasse miraculeusement tous les liens et particulièrement celui qui la reliait à son fils. ${ }^{18}$

A leurs yeux, ce temps ravive l'instant qui s'est écoulé, et capte ce qui leur avait échappé. En fait, les personnages de Seigle, amateurs du passé, aiment posséder les vieux objets qui conservent l'odeur d'antan et évoquent l'ancien temps.

Martin R, Schaïr a souligné que "l'objet peut séduire, raviver des souvenirs, évoquer un savoir, inciter à la réflexion; ils acquièrent ainsi une nouvelle dimension" 19

Ainsi, selon le brocanteur Job, le passé est restitué par les objets anciens. Si «Albert ne voyait dans les objets du passé que la survivance de ses souvenirs d'enfant, alors que pour Monsieur Job le passé, à travers «les antiques choses » comme il nommait

17 Jean-Luc- Seigle, Op.cit. p.134

18 Ibid. p. 68

19 Martin $\mathrm{R}$, Schaïr, La relation homme- objet exposée : théorie et pratique d'une expérience muséologique, Publics et Musée, 1999, volume 15, numéro 1, p 31-43 (http://www.persee.fr) 
les objets anciens, était une manière de lutter contre ce séisme annoncé du monde moderne $»^{20}$

Ces médiateurs d'autrefois sont un moyen de se lancer dans un temps historique d'où émergent une fracture définitive d'avec le monde moderne et une valeur mystique, et font naître des émotions suscitées par le charme du passé.

Lorsque le partenaire d'Albert médite sur les antiquités, il invite la mémoire à effectuer un voyage hors du temps où il navigue dans un flux de réminiscences illimitées. Emilie Charlet souligne que «l'objet fonde une communauté de souvenirs et une mémoire collective" ${ }^{\text {21 }}$

A ce titre, la brocanterie était pour Job «une sorte de purgatoire ${ }^{22}$ qui atteste un temps sacré et historique. Grâce aux antiques, il est apparemment possible de capter le passé, qui sera concrétisé dans leur apparence et d'empêcher les impératifs du monde moderne d'abolir les souvenirs.

Selon Catherine Huvelin, «conserver ces souvenirs donne l'impression que le passé est toujours là, que l'on n'a pas

20 Jean-Luc- Seigle, Op.cit. p.80

21 Emilie Charlet, Op.cit.

22Jean-Luc- Seigle, Op.cit. p.81 
abandonné celui que l'on était. Posséder sert à se prouver sa valeur, son pouvoir. $»^{23}$.

De là, les personnages de Seigle ne respectent pas la linéarité temporelle, et renoncent à la trinité du temps. Albert déclare: «et puis comment être de tous les temps? C'était impossible $»^{24} \mathrm{Il}$ refuse la succession des temps et voit que le passé reste la seule période qui mérite d'être vécue.

Pour cette raison, il a installé près de sa maison un appentis des réveils cassés qui fascinent Albert car, une fois que l'aiguille ne fonctionne plus, il ne ressent plus l'écoulement du temps.

Ces objets usés ne traduisent plus la rapidité de l'instant, mais à l'inverse, ils deviennent le rythme de ses souvenirs figés dans sa conscience. "D'après lui, seule l'horlogerie était capable d'un tel prodige $»^{25}$, il ignore ainsi le présent, ce temps ordinaire représenté par l'aiguille et les calendriers.

En effet, dans le réveil, Albert voit non pas le temps, mais un simple appareil : «lui qui s'obstinait à ne voir dans ses pendules et ses réveils que de simples et fascinants

23 D’après Emilie Gruyelle, Psychologie, Article : " Je Garde tout », 2008, p.1 en (http://www.psychologies.com/Moi/Seconnaitre/Comportement/Articles-et-Dossiers/Je-garde-tout) 24 Jean-Luc- Seigle, Op.cit. p.133 25 Ibid. p..20 
mécanismes ${ }^{26}$ Selon lui, le temps n'est pas une progression; il est intemporel et immobile.

Par ailleurs, vivre le passé consiste également pour ces personnages à refuser tout ce que suggère l'actualité comme nouveauté. Le refus du quotidien se manifeste lors du remembrement des terres agricoles, qui sont un signe du développement du pays par De Gaulle.

Albert s'explique: «Après, ils pourront tout effacer avec leur remembrement, leurs machines à laver le linge et leur téléviseur." "Je ne veux pas être témoin de la fin de ces temps que j'ai tant aimés. $»^{27}$

Exploiter les terres avec des techniques modernes en réduisant le temps et l'effort des agriculteurs en vue d'augmenter fortement la production n'est alors, selon Albert comme selon son partenaire M. Job, qu'une manipulation de l'ancien système.

Les personnages voient dans la modernité un renoncement au passé, une déclaration des temps nouveaux: «Le monde avançait, (...), mais Albert ne voulait plus avançait avec lui $»^{28}$, ils sont sclérosés dans l'ancien système agricole.

26 Ibid. p. 27

27 Ibid. p. 195

28Jean-Luc- Seigle, Op.cit. p.137

-58- 
Ainsi, Albert se montre conformiste lorsque le mot «remembrement» lui laisse entendre que le monde de ses ancêtres a été un monde démembré, sans structure, une espèce de grand cadavre aux membres éparpillés, qu'il ne faut surtout pas reconstituer et auquel il faut redonner une autre forme, plus homogène, dans un ordre plus simple, plus productif, un monstre en somme. Il sent qu'il est seul dans un monde insurmontable, qu'il voit injuste et traître.

Dans son désarroi, Albert rejette le présent, subit des périodes d'angoisse, de colères intolérables et de sanglots inconsolables. Il est conscient de ce qui se passe en lui et hors de lui, et revient à ses sensations et à ses émotions chaque fois qu'il se souvient. Il devient, par conséquent, pessimiste et enfermé.

D'un autre côté, le héros de Seigle ne profite pas du présent pour regarder la part positive du quotidien. Il ne s'intéresse pas au fait d'être payé par son usine et de vivre sous la protection de l'entreprise Michelin. Il préfère revenir à sa vie primitive, et gagner sa vie à travers sa terre, ses volailles et sa petite vigne.

Les aspects de la modernité et le système de la vie actuelle sont pour Albert un phénomène redoutable qui traduit l'insécurité, ce qui l'empêche de s'engager et de participer aux 
avis des autres. Il crie «Le remembrement n'allait pas seulement tuer le souvenir, il allait effacer les traces du passé ${ }^{29}$

Selon lui, vendre ses terres à l'État est un acte haïssable. Il évite tout contact avec le réel, et proteste contre une décision qu'il voit comme déprimante. Ces propriétés font partie de sa vie, et il tente de les protéger.

Heidegger affirme que l'homme possède «ses possibilités d'existence essentielles que porte encore avec lui l'être qu'il fut (l'être-été) sur le mode du "pouvoir être $»^{30}$ donc le passé fait partie de l'existence d'Albert et constitue son pouvoir.

Cependant, Albert vit l'ancien temps qui lui fait ressentir son existence, même si les rappels le font souffrir. Il s'accroche au passé et revoit les douleurs des Français, l'héroïsme qu'éprouvent les résistants devant l'infamie des camps de concentration et des chambres à gaz lors de la seconde guerre mondiale.

Il se rappelle également ses sentiments gênants quand il était soldat prisonnier, la peine qu'il a ressentie à cause de son éloignement de sa maison. Il se culpabilise de la défaite française contre les Allemands et revoit les sarcasmes du monde sur la

29 Jean-Luc- Seigle, Op.cit. 136

30 Martin Heidegger, Etre et Temps, par François Vezin, Gallimard, Paris, 1990, p 267 
reddition des soldats à Schoenenbourg sur la Ligne Maginot où il avait été muté.

Ses pensées s'enfuient dans ses larmes. Il est pris de tremblements et de frissons lorsqu'il se souvient de sa déportation et des années de guerre, phénomènes allant jusqu'à «l'anesthésie totale de tous ses membres » ${ }^{31}$

À travers les entrelacements constants du flux de sa conscience, il est plongé au cœur du temps éclaté devant ces êtres vaincus et défaits dans la souffrance, la violence et la dérision, constamment secoué par ce sanglot si difficile et si ancien. L'air lui manque, ses émotions l'affaiblissent, il se croit presque mort.

Cet état d'affaiblissement est également ressenti par la mère Morvandieux qui se tue dans la tristesse et le souvenir. Elle maigrit, vieillit: "tous les vendredis depuis plus de quarante ans, elle se rendait au cimetière sur la tombe de son fils unique mort au champ d'honneur. ${ }^{32}$

Elle se retire entièrement de la vie, et vit dans les mauvais souvenirs de la mort de son fils. Quelque chose s'est arrêté en elle. Le souvenir de cette période de sa vie qu'est la guerre reste comme un nœud qui étrangle sa mémoire.

31 Jean-Luc- Seigle, Op.cit. p.105

32 Jean-Luc- Seigle, Op.cit. p.103

$-61-$ 
Elle est devenue une des veuves de la première guerre mondiale. Elle vit à moitié morte. Elle est "défigurée », « rabougrie », «fripée », et sombre dans ses réminiscences terrifiantes, à moitié morte entre le fantasme de son mari et celui de son fils jamais revenu : «La mère Morvandieux, elle qui vivait cloîtrée dans ce tête-à-tête avec son fils de dix-neuf ans mort au champ de bataille ${ }^{33}$ qui devient un spectre qui hante sa vie.

Le philosophe Simon Manon a affirmé que : «A divaguer hors du temps de la vie effective, on diffère le temps de vivre, on remet à plus tard le temps d'exister et on oublie d'être. Comme si nous avions le temps de différer le temps de vivre. "134

Cet état de bouleversement vient du fait que le personnage ne vit pas un temps linéaire où un présent chasse le passé et où l'oubli atténue la mémoire des souffrances de jadis. Albert est à l'image de son corps qui, loin de se ramollir avec l'âge, se noue et se durcit de plus en plus parce qu'il se laisse écraser par les rappels de son temps, qui le laissent impuissant pour affronter le présent :

33 Ibid. p. 50

34 Simon Manon, Article : Visage de la folie Humaine, Nous ne tenons plus au présent Pascal, Philolog, Cours de philosophie, 2009. En (http://www.philolog.fr/visage-de-la-folie-humaine-nous-ne-nous-tenonsjamais-au-temps-presentpascal/) 
«Des larmes à nouveau affluèrent sous ses paupières, à nouveau les brûlures dans les yeux, même si ce n'étaient que des larmes d'impuissances. Enfermé dans ses pensées autant qu'il se sentait enfermé dans ce corps inutile. $»^{35}$

Vivre le passé douloureux et nier le présent affectent son physique et son moral. Autant de fois il le remémore, autant de fois il ne se trouve plus comme être vivant parce qu'il ne réagit pas au quotidien. Plus d'existence, plus d'identité : tout s'éparpille dans le néant du passé. Alors, Albert perd son identité et son existence au présent.

Heidegger souligne à ce propos que la sortie du Dasein de la «quotidienneté déchante », de l'emprise du «On» ${ }^{36}$ demande dès lors un véritable arrachement qui ne peut se réaliser que selon deux événements capables de le soustraire au mouvement affairé de l'aliénation rassurante : l'angoisse et l'anticipation de la mort.

De là, renonçant à vivre l'instant, il n'a qu'à renoncer à sa vie, comme le souligne le grand philosophe. Albert attend la mort pour mettre fin à son chagrin, et ne laisse pas le présent qui s'écoule abolir son passé.

35 Jean-Luc- Seigle, Op.cit. p. 33

36 Notons que "Le " On " selon Heidegger est une personne sans être, sans quotidien et sans responsabilité qui l'attache au monde séparé de tout contact naturel. 
Albert veut mourir et souhaite que sa mort, à défaut d'être une fin, ressemble à un rêve qu'il aurait réalisé. Vivre le présent n'est qu'une menace pour Albert, et ce qui le rassure est la mort à travers laquelle il fuit la fatalité de ce destin.

Heidegger considère que le point de flexion entre l'angoisse et l'anticipation de la mort est soit se perdre dans le monde, soit se ressaisir en tant que soi-même. ${ }^{37}$ Or la mort garantit à Albert d'exister dans le néant, d'être enseveli parmi ses ancêtres et de sauvegarder son passé intime, qui est la marque de son existence.

Le grand philosophe interprète ceci à ses mots : «le Dasein existe toujours, projeté dans cette possibilité et donc en avant de lui-même, décalé, "libéré de toutes les familiarités et les affairements du quotidien, il entraîne tout avec lui dans le néant ${ }^{38}$

C'est dans le néant que les amateurs du passé trouvent le repos. Vivre dans la mort reste un moyen de pouvoir être. Albert conçoit que la mort fait partie de son existence et affirme que les hommes s'éteignent pour naître dans la mort. Le terme « empurger » apaise ainsi Albert et de sa mère.

37 Cf. Martin Heidegger, Etre et Temps, par François Vezin, Gallimard, Paris, 1990, p 46-60

38 Michel Haar, Martin Heidegger, Le concept de temps, Cahiers de l'Herne, $N^{\circ} 45,1983$, p. 53. En (https://fr.wikipedia.org) 
Le couple mère-fils a envie de mourir et de rejoindre ses ancêtres. "l'Empurgar et toute la mythologie enfantine du ciel chrétien où se réfugient les morts réveillèrent son désir d'en finir (...). Plus que jamais Albert eut envie d'être "là-haut »"

Alors, Albert devient pessimiste et enragé tant que la fin est en retard. Il anticipe ainsi sa fin comme l'a expliqué Saverio Tomasella :

«Tant que cette rage n'est ni acceptée ni reconnue par l'entourage (et par soi), elle se répète et se reproduit dans des mises en acte destructrices envers les autres ou soi-même. La fureur désespérée peut devenir meurtrière ou suicidaire. ${ }^{40}$

Angoissé, il décide de s'ensevelir avec ses souvenirs et de quitter définitivement le présent, qui lui arrache le passé. Il anticipe sa fin par le suicide pour être enterré avec son passé. Heidegger a affirmé que dans le néant, l'homme peut s'éprouver lui-même comme possibilité, comme "pouvoir-être ", au lieu d'être perdu dans les méandres des temps.il affirme que «L'être, c'est donc tout simplement le néant $»^{41}$

39 Jean-Luc- Seigle, Op.cit. p. 170

40 Saverio Tomasella, Le sentiment d'abandon, Eyrolles, Paris,2010, p. 33

41 Franz- Emmanuel Schürch, L'Etre, l'étant, le Néant, Revue de Métaphysique et de Morale, presse universitaire de France, no3, 2008 (https://www.cairn.info) 
Nietzsche interprète l'homme qui vit sans oubli comme étant perdu dans un monde labyrinthique, il signale qu' : «un homme qui serait incapable de ne rien oublier et qui serait condamné à ne voir partout qu'un devenir; celui-là ne croirait pas à sa propre existence, il ne croirait plus en soi, il verrait tout se dissoudre en une infinité de points mouvants et finirait par se perdre dans ce torrent $d u$ devenir $\gg .{ }^{42}$

À cet égard, le héros postule sa fin anticipée pour se déplacer dans un autre état où il existe et se protège du présent chaotique : "Maintenant, il ne voulait plus que les morts autour de lui, la longue généalogie des ancêtres dans la terre dont il reconnaissait l'haleine quand la brume se levait sur les champs aux crépuscules ${ }^{43}$.

Albert choisit cet état d'immobilité où il évite un temps irréversible, qui chasse son passé intime et le perd dans ses tourbillons. Vivre la fin représente une victoire sur le temps pour Albert puisque l'homme décide de sa fin et de sa vie à l'abri d'un temps tyrannique et autoritaire.

Le personnage refuse d'être le jouet du temps et décide de choisir son destin, de vivre un temps illimité et indéfini où l'âme ne souffre plus et où l'homme perpétue son passé : l'origine de son être. Proust a précisé que la mort est une autre vie mais hors

42 Henri Albert, Frédéric Nietzsche. Considérations inactuelles. T. 1 Mercure de France, Paris, 1873, p.126 en (http://gallica.bnf.)

43 Jean-Luc- Seigle, Op.cit. p. 196 
du temps: "l'autre vie, celle où on dort, n'est pas - dans sa partie profonde - soumise à la catégorie du temps ${ }^{44}$

Cependant, en choisissant le passé, le personnage du récit ne jouit plus de sa vie quotidienne, puisqu'il est figé d'une part par la claustration d'un temps vécu, d'autre part par la peur de l'instant menaçant. Alors, vivre le seul temps présent donne-t-il accès au bonheur?

\section{Le refus du passé}

44 Marcel Proust, $A$ la recherche du temps perdu, Gallimard, Paris, 1919, p 154

$-67-$ 
Certains personnages conçoivent le passé comme un ennemi à combattre. Le passé trouble l'instant et transforme la joie de vivre en angoisse. Ils suivent ce que le philosophe André ComteSponville a expliqué :

" Je suis mon corps actuel, mon corps en acte, et cette matérialité de mon existence n'est pas autre chose que ma présence au monde - ma présence au présent. $\gg 45$

De ce point de vue, Suzanne, l'épouse d'Albert, refuse le passé qui perturbe l'instant, et croit que vivre l'actualité est un moyen d'échapper aux réminiscences de jadis.

Elle se distrait en se remémorant ses souvenirs, en pensant à son plaisir, aux habits qu'elle portera et aux lettres qu'elle écrit : «ses petites choses lui permettaient de s'extraire du monde des autres, de plonger en quelques sorte dans le vivant ${ }^{46}$

Elle renonce à se replier sur elle-même malgré le départ de son fils Henri qui risque sa vie dans les émeutes en Algérie, affamé sous le soleil torride. Elle ne s'enfonce pas dans son rôle de mère douloureuse, et ne s'effondre pas à l'évocation de son enfant chéri. Elle se redresse, retouche son maquillage, et se remet à table pour vivre son quotidien.

Elle s'oublie également dans la danse jusqu'à la transe, s'enivre dans la natation, se réjouit de la légèreté du contact de

45 André Comte-Sponville, L'Etre-Temps, Puf, 1999, p. 133 46 Jean-Luc- Seigle, Op.cit. p. 57 
son corps avec l'eau. À ses yeux, le passé n'existe plus, elle ne pense pas non plus à l'avenir. Sa conscience évite de conserver ses impressions douloureuses du passé, et refuse de planifier l'avenir. Elle change continuellement d'image, entre celles de mère, d'épouse et de femme. Suzanne crie son refus «Le passé, ça sert à quoi? ", elle "s'acharnait à en effacer toutes les traces. ${ }^{47}$

Afin de vivre le présent, elle prend d'abord du recul par rapport au flux continuel des souvenirs : elle oublie ses années d'orphelinat avec les religieuses, où elle était négligée; elle renouvelle son monde sur les décombres du passé :

«Suzanne mettait tous ses espoirs dans un monde qui, justement, n'avait jamais existé avant, un monde de perfection, à la construction duquel elle tenait à participer avec la plus grande dévotion. $»^{48}$

Elle suit le principe que déclare Albert camus que «le présent est la seule dimension temporelle où l'homme puisse être heureux $\gg 49$

47 Ibid. p.96

48 Ibid. p.40

49 Maurice Weyembergh, Albert Camus, ou, La mémoire des origines, De Boeck université , Paris, 1998, p. 159 
À la recherche du bonheur, elle s'éloigne de son époux qui s'accroche aux traditions campagnardes du passé. Elle refuse la vie primitive qu'il lui impose : celle d'épouse d'un paysan.

Elle renonce à avoir les ongles noirs, à transpirer comme un bœuf et à sentir l'odeur des vaches et du fumier. Elle est contre l'élevage des bêtes et pour l'achat de la viande à la boucherie. Elle s'efforce de s'approvisionner à la charcuterie pour satisfaire tous ses besoins nutritifs :

«Pour Albert ce fut le premier signe de résistance que sa femme opposa au principe qu'il avait toujours déclaré: la nourriture, ça ne s'achète pas. » ${ }^{50}$

Dans cette optique, elle vit le présent avec ses progrès, et l'applique au quotidien. Elle adopte en outre une stratégie de renouvellement continu dans ses pratiques journalières, ses idées et son allure. Elle s'inscrit dans le changement constant, anéantit tout ce qui est ancien et suit la modernité : «Moderne était le seul mot auquel Suzanne se référait après Dieu » ${ }^{51}$.

Alors, elle s'obstine à transformer en maison moderne la ferme où elle est logée et où son mari est né : "Crépir au ciment les murs extérieurs de la ferme et les avoir fait repeindre en

50 Jean-Luc- Seigle, Op.cit. p. 20

51 lbid. p.39 
blanc, coffrer les linteaux, remplacer les fenêtres, rien n'avait l'air neuf. " ${ }^{52}$

Cette maison, qui était à ses yeux un trou et une léproserie troglodyte datant $\mathrm{du}$ XIII ${ }^{\mathrm{e}}$ siècle, est devenue propre. Elle renouvelle les différents objets présents dans la maison, comme les torchons qui, bien que reprisés, témoignent du soin que Suzanne apporte aux choses neuves.

Plus tard, elle renouvelle la salle à manger qui était de style Henri II en enfilade scandinave complétée de six chaises en fer forgé qu'elle a achetées. Elle apporte à son domicile une touche moderne en l'équipant d'un poste de télévision qui rompt la vie monotone de la maison Chassaing.

Elle construit ainsi une vie moderne en installant de nouveaux objets comme "cuisine, chambre placage acajou en kit, cosy chambre Henri, moulin à café électrique, frigidaire, $4 C V$, planche à repasser pliante $»^{53}$ en plus "poste de télévision $»^{54}$. Ceux-ci sont les témoins de la technologie contemporaine et de la mode qui lie l'héroïne au présent, la pousse à s'infiltrer dans la vie moderne et à s'en réjouir.

52 Ibid. p.40

53 Ibid. p.77

54 lbid. p.77 
Le présent envahit sa vie au détriment du passé, mais ce temps est fugace, aléatoire. Alors, Suzanne immortalise les nouveaux objets, comme les ustensiles de la vie moderne. L'inventaire d'appareils électroménagers neufs marque un nouveau temps. Elle construit par le biais de ses objets un album qu'elle qualifie de projet de construction de vie moderne.

Repoussant le passé, elle n'est pas attirée par le charme que suscitent les objets antiques, mais s'intéresse plutôt au mobilier contemporain, préférant une table moderne en formica plutôt qu'une antique porcelaine chinoise. Le narrateur affirme son dégoût en affirmant :

«Elle détestait ce mobilier tarabiscoté et espérait bien l'échanger contre une enfilade scandinave et six chaises en fer forgé qu'elle avait repérées (...) des Galerie de Jaude ${ }^{55}$

De là, elle procède à une série de ventes sans fin au nom du changement et d'une vie meilleure. Elle vide la maison des cadeaux des grands-parents, de ses anciens amis, de la salle à manger du siècle passé, et de l'ancienne bergère de sa belle-mère.

Cette modernité luxuriante annonce le présent pour Suzanne. Cette nouvelle apnée dans l'âge moderne s'annonce

55 Jean-Luc- Seigle, Op.cit. p. 41

$-72-$ 
lorsqu'elle vend en plus les objets et meubles anciens au brocanteur pour faire table rase d'une vie primitive monotone.

Bergson souligne qu'«il ne s'agit pas d'atteindre une conscience de la fin de l'être pour saisir l'être même, mais au contraire de vivre dans le flux continuel de la nouveauté. ${ }^{56}$

En effet, Suzanne s'efforce de suivre la mode, et prend certaines habitudes précieuses d'une femme moderne comme « la toilette du soir ». Elle aime paraître toujours bien maquillée et bien coiffée. Elle change d'allure vestimentaire en renonçant définitivement aux robes-tabliers informes et sans style qu'elle portait. Pour elle, ces habits ne sont pas des signes de jeunesse ou d'élégance. Elle les transforme en torchons, et les remplace par de nouvelles robes.

Afin de parfaire sa métamorphose, elle renonce à ses chaussures plates, et porte des escarpins à talons aiguilles qui donnent un aspect gracieux à ses mollets et à sa démarche. Elle se renouvelle «Suzanne avait passé une nouvelle robe bleue très simple, sans manche et assez près du corps, qu'elle avait confectionnée elle-même dans la semaine ». ${ }^{57}$

56 Tatjana Barazon, L'expérience du temps De la phénoménologie à Bergson, Esprit d'avant, Chronique, No 2, 2008

57 Jean-Luc- Seigle, Op.cit. p. 109 
En fait, son élégance, témoin de son intégration dans le présent, bouleverse l'ancienne image de la paysanne incrustée dans la pensée de son mari Albert. Il la voit en révolte contre les croyances du passé et contre la figure de la fermière en plein labeur avec son mari :

«Albert, lui aussi, avait remarqué l'étonnante métamorphose de sa femme. C'était vrai qu'elle devenait chaque jour de plus en plus belle $»{ }^{58}$

De peur d'altérer sa modernité et sa beauté toujours renouvelées, elle s'attache à la photographie, qui inscrit dans le présent le renouvellement de son aspect. «Elle était prête pour se faire photographier, escarpins blancs, robe bleu ciel, les cheveux coiffés et les yeux peints, son sac à main en cuir blanc ${ }^{59}$

Véronique Gocel reprend à ce sujet que «Pour ainsi dire nier le temps, donnant l'illusion que la photographie est un de ces instantanés, une de ces coupes lamelliformes pratiquées à l'intérieur de la durée et où les personnages aplatis, enfermés dans des contours précis, sont pour ainsi dire artificiellement isolés de la série des attitudes qui précèdent et qui suivent. ${ }^{60}$

58 Jean-Luc- Seigle, Op.cit. p. 48

59 lbid. p.147

60 Véronique Gocel, Histoire de Claude Simon : écriture et vision du monde, Peeters, Paris, 1996, p 111. 
La photographie lui permet alors de capter ces instants fugitifs, et d'enregistrer ses meilleurs moments pour qu'ils ne retournent jamais au passé. Suzanne s'oublie en se distrayant et en s'impliquant dans le quotidien: «Il lui suffisait de penser à la mise en plis qu'elle devait se faire, à la robe qu'elle allait porter, à la prochaine lettre qu'elle écrirait pour que tout commence à aller mieux ${ }^{61}$

En outre, elle modifie son ancien accent patois, et s'efforce de parler un français parfait et soutenu. Liliane, sa belle-sœur, partage avec elle cet amour de la nouveauté «aimait voir le passé enfermé dans une vitrine ${ }^{62}$. Ainsi, elle parle comme Suzanne le français contemporain :

«Parler patois serait perdre tout ce qu'elle avait essayé de conquérir dans cette France nouvelle dont elle partageait les promesses avec ses amies aux "femmes françaises 》 et avec Suzanne ${ }^{63}$

À ses yeux, la vie moderne abolit le passé austère et ravive l'esprit: «elle (Suzanne) était convaincue que la vie moderne était la meilleure réponse à ses prières, après les années de

61 Jean-Luc- Seigle, Op.cit. p. 56

62 Ibid. p. 108

63 Ibid. p. 107

$-75-$ 
privations et d'efforts qu'elle avait endurées pendant la guerre et à la libération ${ }^{64}$

Ce temps moderne passionne les amateurs du présent, partisans du progrès et avides d'originalité. Suzanne, comme sa belle-sœur Liliane et son époux André, croit en cette idée moderne qu'est le remembrement des terres agricoles qui va de pair avec la reconstruction de la France. Tous trois sont favorables à la construction de ponts hydrauliques qui les rendent si fiers.

En revanche, ils oublient complètement l'ancien temps, qui ne leur rappelle que les misères auxquelles les hommes étaient soumis. Le passé reste à leurs yeux le temps de la décadence, le témoin d'une vie infernale :

«Ce fut donc avec la plus grande application et la plus grande dévotion qu'elle (Suzanne)se mit à détruire le monde d'avant-guerre pour tenter d'y rebâtir un monde nouveau. ${ }^{65}$

Par conséquent, leur croyance rompt avec les idées anciennes comme la féodalité du Moyen Âge, et accueille de nouvelles idées comme le communisme et le développement social.

64 lbid. p.39

65 Jean-Luc- Seigle, Op.cit. p.39 
Certains personnages de Seigle renient leur passé, et vivent sans racines en lutte perpétuelle contre un temps ennemi. Aucune attache familiale ne leur rappelle d'où ils viennent. Lorsqu'ils se souviennent des événements passés, ils ressentent une certaine amertume. Ils ont besoin d'un renouvellement pour ressentir leur existence.

De ce point de vue, Suzanne instaure des changements dans tous les aspects de sa vie, y compris au niveau des personnes qui l'entourent. Alors, elle oublie la présence de son mari, et se lance dans une relation extraconjugale à la recherche du plaisir charnel. En commettant l'adultère, elle s'oublie dans l'instant :

«Elle avait attendu toutes ces années de femme pour oublier l'épouse et la mère qu'elle avait été jusqu'à cet instant, pour ne plus être qu'une femme, un désir, une peau, s'oublier au point de ne sentir que son corps de plaisir,(...) ${ }^{66}$ Elle s'extasie dans l'instant exubérant, et s'affole dans son contact avec la nature.

Elle néglige également son fils, en s'occupant d'elle-même sans tenir compte de sa présence. Après la mort de son mari, elle abolit toute trace de son passé : elle vend la maison conjugale, et se marie avec un autre. Suzanne aspire à changer sa vie à la

66 Ibid. p. 160 
campagne. Elle souhaite s'installer en ville, et redevenir une inconnue.

Plus tard, elle a changé de domicile «la propreté était la preuve éclatante et incontestable de son engagement dans la vie moderne qui la conduirait, un jour, à fuir ce trou pour aller vivre dans un pavillon (...), rempli de lumière, au dernier étage d'un immeuble neuf. ${ }^{67}$

Robert Paris souligne à ce propos que «L'image que nous avons de nous-mêmes est déterminée par notre passé et le passé permet "de tisser un lien essentiel entre notre "moi d'hier" et notre "moi d'aujourd'hui"..» ${ }^{68}$

Cependant, le personnage se rebelle de plus en plus contre sa vie monotone. En tant qu'épouse Chassaing, elle s'est rendue plus menaçante que rassurante. Elle veut renouveler tout ce qui l'entoure. Or, l'héroïne se trouve en état de changement continu qui s'impose tous les jours en vue d'empêcher l'instant présent de devenir un passé.

67 Ibid. p. 40

68 Robert Paris, Matière et révolution, La mémoire, une reconstruction du passé tournée vers le futur, article 497, 2008, en (http://www.matierevolution.fr/spip.php?article497) 
Donc, Jacques Derrida affirme que dans cette désarticulation et ce changement continuel «Le temps fissure la possibilité d'une simple identité à soi ${ }^{69}$.

De là, l'héroïne n'a pas un passé alors que son identité risque de s'égarer comme l'a repris Roland Gori : il n'y a plus d'hier, donc plus d'identité.

Il interprète cet état d'oubli de l'histoire de notre vie comme « un déficit cognitif (...), un échec de récupération des données du passé. $»^{70}$, Robert Paris ajoute à ceci que «Sans mémoire, l'être humain ou l'être vivant perdrait tous ses repères et toute conscience de son identité. » ${ }^{71}$

De là, l'héroïne n'a pas de passé. Son identité risque alors de s'égarer: il n'y a plus d'hier, donc plus d'identité. Ce que Suzanne ressent, c'est l'angoisse de l'égarement, mais en même temps le plaisir de l'instant.

Dans cette turbulence, la douleur, comme le plaisir qu'elle ressent encore, finit de l'exclure du reste du monde. Esseulée et

69 Jacques Derrida, La Voix et le Phénomène, PUF, Paris, 1967, p73

71 Roland Gori, La mémoire freudienne : se rappeler sans se souvenir, en

Cliniques méditerranéennes, éd. Eres, $\mathrm{n}^{\circ} 67$ (2003), p. 2 (https://www.cairn.info)

72 Robert Paris, Op.cit.

73 Maurice Weyembergh, Op.cit. p.161 
inconnue, elle ne participe pas à la vie des autres, alors qu'elle est perdue dans son propre monde, sans ancrage.

«Faute du souvenir des événements passés, le présent serait sans racines et sans épaisseur; privé de l'imagination et de l'anticipation des possibles, il serait sans ouverture et sans perspective. $\gg^{72}$

Par conséquent, elle vit dans un regret continu : le présent lui est agréable, et pourtant, il lui échappe. Chaque instant du présent devient plus tard un passé, ce qui la rend fatiguée des changements consécutifs pour capter l'instant éphémère.

En fait, elle vit un temps indéfinissable, insaisissable, qui n'existe que dans la fuite, qui n'apparaît que pour disparaître. Son instant est fugitif, ne se révèle qu'en se dérobant, ne se donne que dans sa perte.

De ce point, Friedrich Hegel, dans La phénoménologie de l'esprit, affirme à propos du présent fugitif que : "Le maintenant est justement ceci de n'être déjà plus quand il est." ${ }^{73}$ Il lui semble que cet instant ne peut pas être vécu puisqu'il a déjà disparu au moment où l'on en prend conscience.

74 Friedrich. Hegel, Phénoménologie de l'esprit, Tome I, Traduction de Jean Hyppolite, Montaigne, Paris,1807, p. 88 (https://books.google.com.eg) 
Sans passé, on ressent l'écoulement rapide du temps. : «Un monde sans passé est aussi un monde sans présent, s'éternisant dans le non-temps. » ${ }^{74}$ Comme le souligne Saint Augustin « Si rien n'était, il n'y aurait point de temps présent. ${ }^{75}$

Alors, si le personnage de Seigle ne trouve jamais le plaisir de vivre dans le passé ou dans le présent, comment peut-il se réjouir?

\section{La jouissance du temps}

Si certains personnages du roman de Seigle s'acharnent contre le passé ou le présent, les considérant comme deux ennemis à combattre, d'autres échappent à ce pouvoir du temps qui cause soit l'anticipation de la mort comme pour Albert, soit la perte de soi-même comme chez Suzanne. Ils décident d'arrêter ce conflit et de renouer avec les deux temps en les vivant alternativement.

En fait, Gilles, le fils d'Albert, reconnaît leur linéarité, et souligne qu'il est indispensable de s'intégrer et d'éviter tout écart. Il déclare que «le passé, s'il est connu, n'est connu qu'à

74 Thi Tu Huy NGuYen, La néantisation du temps chez Robbe-Grillet, Revel revue électronique, Université Nice, paru en Loxias, 2008, no 22.

76 Jean Alexandre Buchon, Choix d'ouvrages mystiques, dans "Confessions de St -Augustin »- Livre XI, Ed. A.Desrez, 1840, p.185 77 Jean-Luc- Seigle, Op.cit. p.71 
travers le medium du présent ${ }^{76}$, comme le signale Etienne Klein auparavant :

«Une deuxième difficulté vient de ce que nous ne pouvons pas nous mettre en retrait par rapport au temps, comme nous ferions pour un objet ordinaire. Nous pouvons le mesurer, mais pas l'observer en le mettant à distance, car il nous affecte sans cesse. Nous sommes inexorablement dans le temps. $\gg{ }^{77}$

Alors, se réconcilier avec le temps reste une passion pour le maître d'école de Gilles. Il s'agit pour lui d'une idée qui s'impose sur la vie et qui fait partie de son existence. Il confirme cette coexistence des deux éléments en soulignant que l'histoire fait partie de sa vie.

Il confirme cette coexistence des deux éléments en soulignant que l'histoire fait partie de sa vie «elle vit avec nous, même si on reste sur place toute sa vie. «(...), elle finit toujours par s'asseoir à notre table. ${ }^{78}$

À chaque instant dans le présent, on se rappelle des moments dans le passé. Par conséquent, les deux temps ne sont ni

78 Michel Cazenave, Etienne Klein, Dictionnaire de l'ignorance, « Le temps de la physique », Albin Michel, France, 1998, en Centre international de recherches et études transdisciplinaires (CIRET).

79 Jean-Luc- Seigle, Op.cit. p.95

80 Christophe Bouton, Temps et esprit dans la philosophie de Hegel : de Francfort à léna, Vrin, Paris,1991, p.96 
étrangers ni opposés, car l'homme voit le passé dans le présent, et vice versa.

Afin de les faire coexister, les personnages recourent à des éléments médiateurs de la transmission du passé dans le présent. $\mathrm{Ce}$ sont les témoins d'un passé lointain qui se manifestent concrètement sous leurs yeux. Ces témoins s'engagent à aider les personnages à remonter dans le temps et à établir une proximité.

Ainsi, les personnages tendent à revivre un instant unique d'abord par le biais des objets d'art: André Malraux souligne que «ce qui compte de l'art du passé est présent(...), il est dans son éternité, il est dans son temps historique ou chronologique, et il est dans le présent $\gg{ }^{79}$

En effet, ces éléments concrétisent la correspondance des temps, comme ceux du maître d'école. Ainsi en est-il des tableaux de la guerre datée de 1870 : trois gravures sous verre représentant des personnages antiques de la mythologie grecque, comme Ulysse, Télémaque ou Athéna, rassemblent toutes les empreintes du passé, mais en plus, ils sont constitutifs d'une histoire passée que la scène se charge de faire advenir à nouveau.

79André Malraux, Lazare. Le Miroir des Limbes. Gallimard, Paris, 1974. p.322

$-83-$ 
L'objet cristallise l'introspection, et devient un lieu mémoriel qui ravive l'esprit et établit une liaison simultanée entre les deux temps. Martin R. Schärer souligne que :

«La beauté des objets interpelle directement le visiteur plongé dans la contemplation et fait naitre en lui une sensation unique. (...), il retrouve enrichi son quotidien $»^{80}$

La présence du passé dans le présent abolit le temps puisque les objets représentant le passé deviennent témoins de l'actualité, en même temps qu'ils bouleversent l'irréversibilité du temps. Ces objets évoquent un flux de souvenirs et d'images ressuscités au présent.

Ainsi, Gilles vit constamment un présent « réflexif » qui se double avec le temps passé. Dans cette optique, Roland Barthes souligne que «l'objet se fait mémoire visible et manipulable d'un état du monde et d'une histoire, un supplément d'âme

qui rend le passé évoqué beaucoup plus limpide, qui investit la matière inerte, un objet évocateur qui marque une vie et une histoire. ${ }^{81}$

Cette méditation sur le passé à travers le présent empêche de ressentir l'écoulement du temps du fait qu'on fait arrêter ce

80 Martin R. Schärer, Op.cit. p.36

81 Émilie Charlet, Op.cit.

-84- 
dernier à chaque fois qu'on médite sur l'œuvre artistique. La restitution du passé dans le présent idéalise l'instant vécu et anime l'esprit.

D'ailleurs, vivre l'homogénéité du temps peut également se faire par le biais des livres qui évoquent une ancienne époque, considérés comme des représentants d'un passé lointain. Ils sont des points d'accès entre le présent et le passé, et permettent aux personnages de vivre un instant glorieux grâce à la lecture de textes de siècles passés.

À travers les œuvres de grands écrivains témoins d'un passé lointain comme Voltaire, Balzac et Hugo, Gilles change de lieu et de temps et revient un siècle en arrière: «Grâce aux mots de Balzac, à la manière de les agencer en images, il parvenait à sauter dans le décor, sans même se rendre compte qu'il changeait de siècle ${ }^{82}$

Ce mouvement permet l'infusion des temps qui s'enlacent jusqu'à la confusion de toute chronologie, qui crée une profondeur de l'instant présent, riche d'autres temps que le livre fournit à chaque moment.

82 Jean-Luc- Seigle, Op.cit. p.37

-85- 
Gilles procède à cette réconciliation avec son temps en faisant correspondre les paysages évoqués dans Eugénie Grandet de Balzac avec ceux de son environnement :

$\mathrm{Au}$ champ de cerisier qui entoure sa maison au village d'Assys, "Gilles eut envie de reprendre sa marche imaginaire dans les rues sombres de Saumur couvertes de petits pavés caillouteux et humides jusqu'à la maison du père Grandet $»^{83}$

Cette correspondance entre deux lieux et deux moments réciproques lui fait constater qu'ils se ressemblent: "parce que la maison des Grandet ne semblait pas si différente des maisons de ses vieilles voisines, carrées, en pierre noire des volcans, qui gardait les mêmes odeurs austères des cendres froides, de la cire et du Miroir des cuivres ${ }^{84}$.

Cette affinité entre deux demeures est renforcée par un autre rapprochement de type sonore, entre les bruits du jardin de son père qui étaient identiques à ceux du jardin

de la famille Grandet. Par conséquent, il conclut qu'il grandissait dans un monde encore ancien et relativement inerte, comme celui d'antan. Il parvient ainsi à faire coexister deux époques et deux lieux différents : ceux des $\mathrm{XIX}^{\mathrm{e}}$ et $\mathrm{XX}^{\mathrm{e}}$ siècles.

83 Op.cit. p.35

84 Op.cit. p.37

-86- 
En effet, Gilles vit le vrai temps, croit au passé et au présent qui constituent la chronologie naturelle, alors que ce va-et-vient entre les deux périodes de la vie lui procure une sensation de liberté et d'emprise sur les deux durées.

En fait, le personnage se réjouit de bon matin lorsqu'il se lance dans la lecture de ce roman d'antan en quittant son village actuel d'Assys pour s'installer près des invités dans la grande salle de la maison Grandet. Il vit avec eux dans leur demeure par la pensée, mais il se trouve physiquement dans sa maison.

Il voit en même temps le personnage de Nanon qui allume le feu dans la cheminée en présence de Charles, le cousin d'Eugénie, et il est réellement dans sa demeure à Assys, face au cerisier du jardin. A travers la lecture, «Gilles aimait écouter leurs confidences de femmes et en éprouvait un plaisir équivalent à celui qu'il trouvait dans les livres où il découvrait des mondes inconnus, souvent éloignés, mais qui se révélaient à lui comme une autre réalité et l'invitaient à grandir ${ }^{85}$

Cet accès à d'autres lieux permet des correspondances avec d'autres personnages, enrichissant le présent de nouvelles rencontres qui s'ajoutent à celles du quotidien.

85 Jean-Luc- Seigle, Op.cit. p.36

-87- 
De ce fait, le héros bénéficie d'un temps doublé, où il prolonge sa vie d'une autre vie et acquiert une double expérience : celle de la vraie vie et celle du passé. Cet instant magnifique n'est qu'une exploitation du passé en relatant les faits et les paroles des prédécesseurs ainsi que ceux de sa famille et de ses proches.

Il compare les thèmes abordés, les expressions et les réactions des personnages de jadis à ceux du moment réel. Il conclut que les faits et les sujets du passé se répètent au présent. Ainsi, le passé est un référent et un moyen de discerner et d'interpréter le présent.

Martin R. Schärer affirme que «C'est donc exclusivement grâce au présent que l'histoire peut se constituer, elle est une constante réactualisation du passé. ${ }^{86}$

De ce point de vue et à travers Eugénie Grandet, Gilles a pu s'expliquer la métamorphose de sa mère en la comparant à celle d'Eugénie lors de sa rencontre avec son cousin Charles. Le soin qu'Eugénie apporte à son apparence physique pour attirer l'attention de Charles lui permet de comprendre le même soin apparu chez sa mère lors de la rencontre de Paul Marsan, ami de la famille Chassaing.

86 Martin R. Schärer, Op.cit. p.34

$-88-$ 
Il retrouve dans la rapidité du personnage du roman des comportements et des attitudes qu'il avait repérés chez sa mère. Gilles découvre l'amour naissant de sa mère à travers le comportement de l'héroïne de Balzac, deux siècles en arrière.

De même, Gilles comprend la proposition de son père d'étudier avec le maître d'école et le brocanteur comme un changement brusque dans sa vie. Il interprète ce fait à la lumière du père Grandet de Balzac, lorsque celui-ci a introduit Charles chez la famille d'Eugénie, Balzac ayant également considéré cet événement comme un bouleversement dans la vie de son héros Charles.

Gilles explore le présent grâce au passé : à travers les larmes que verse Charles Grandet lorsque son père meurt, Gilles comprend qu'un fils peut pleurer à la mort de son père. Il croyait qu'un homme ne pleure jamais, mais il conclut enfin que pleurer son père mort, quel que soit son âge, doit être normal.

Les événements se répètent, et Gilles compare l'attitude de son père à celle du père Grandet :«Gilles comprit alors que chaque roman qu'il lirait l'aiderait à comprendre la vie, luimême, les siens, les autres, le monde, le passé et le présent, une expérience similaire (...) ${ }^{87}$

87 Jean-Luc- Seigle, Op.cit. p.60

-89- 
D'ailleurs, les mots de Balzac reviennent au secours de Gilles au moment où il découvre le suicide de son père, et les larmes que verse Charles Grandet à l'annonce de la mort de son père s'associent à la souffrance de Gilles : Le roman de Balzac soulage Gilles par ses mots :

«Encore enfant, encore dans l'âge où les sentiments se produisent avec naïveté ». Il maintient son équilibre face à la mort de son père, il avait pour balancier «d'un côté la voix de son père qui murmurait encore son prénom Gilles, «(...), et, de l'autre, tous les mots de Balzac serrés dans sa main. ${ }^{88}$

Le personnage ne sent plus l'écoulement du temps puisqu'il renoue avec lui et que ce dernier lui permet d'abolir les frontières entre le passé et le présent.

Kristeva appelle «temps hors-temps» (...): lorsqu'une perception présente se rapporte à une sensation passé - parce qu'elle est induite par un désir similaire - , la mémoire involontaire la retrouve et suscite l'association de ces sensations ressemblantes et leur métamorphose en une impression,

88 Op.cit. p. 211

$-90-$ 
laquelle, effaçant les frontières des espaces et des époques, du senti et du pensé, brouille leurs identités respectives. ${ }^{89}$

De cette optique, la grande philosophe affirme que ce temps unique ne se réalise que lorsque l'homme vit l'homogénéité du temps.

Alors, grâce à cette incursion dans le passé, «Comme un plongeur sous-marin, Gilles refaisait surface après une longue apnée dans le XIXe siècle ${ }^{90}$, les phrases de Balzac font écho à ce que ressent Gilles et l'aident à exprimer ses sentiments:

«Il avait de plus en plus l'impression que le livre, au-delà de l'histoire qu'il racontait, parlait de lui, comme lui-même n'était pas capable de le faire. C'était étrange et fascinant. Il en jouissait de la même manière que le père grandet jouissait de son or. $)^{91}$

Le siècle précédent répond aux interrogations de Gilles. «Gilles dans un monde de vieux et tirait certains avantages de cette position ${ }^{92} \mathrm{Il}$ crée avec le roman qu'il lit un lien temporel et une proximité presque physique.

89 Julia Kristeva, Marie-Christine Navarro, Au risque de la pensée, L'Aube, 2001, p. 63

90 Jean-Luc- Seigle, Op.cit. p.141

91 Op.cit. p. 142

92 Op.cit. p. 36 
Le livre devient ainsi comme le souligne Christian Jacob : « un lieu où la mémoire fait corps, où la tradition et le passé se matérialisent dans le présent, où la transmission s'inscrit dans un espace de conservation et d'exploitation intellectuelle, mais aussi d'exhibition aux riches effets symboliques. ${ }^{93}$

S'entendre avec le temps permet au personnage de s'infiltrer dans un autre siècle et de s'identifier aux anciens. Gilles donne «à Balzac une existence et une réalité géographique immédiate, une sorte de vieil ami (...) qui avait toujours vécu tout près d'Assys ».94

Le temps l'aide à comprendre les aspects mystérieux de la vie quotidienne. Il se réjouit d'un passé ressuscité qui s'entremêle avec le moment présent. Ce passé, conçu comme perdu, tend à se faire valoir comme perçu.

Alors, le personnage de Seigle perçoit simultanément deux événements réciproques : celui du passé du siècle de Balzac, et celui du présent avec sa famille. De ce fait, il donne à l'ancien temps un aspect instantané, alors que le présent qui doit être fugitif et contingent devient immuable. Le héros bouleverse ainsi la forme canonique et irréversible du temps.

93 Christian Jacob, revue Diogène, Retour vers le Futur, "Rassembler la mémoire », no 196, 2001, p.53

94 Jean-Luc- Seigle, Op.cit. p.86 
D'ailleurs, les personnages de Seigle recourent à d'autres moyens pour vivre un temps similaire et gracieux en liant l'Histoire au présent. Le maître d'école souligne que «l'histoire des hommes, c'est l'inverse de la solitude. Et puis le passé, si nous savons le lire ou l'entendre, nous assure de ce qui est juste ${ }^{95}$

Gilles se réfère aux événements historiques pour justifier et expliquer des phénomènes sociaux de l'actualité. Le passé peut alors être investi par le biais de l'histoire des hommes et guider le présent. Un retour sur le passé et sur l'histoire évite à l'homme d'aujourd'hui de se tromper. Ce va-et-vient permanent entre l'histoire et le présent tisse des expériences de vie déjà vécues et balise le chemin de la vie humaine.

En fait, le maître d'école apprend à Gilles l'importance de ce lien. Alors, pour que Gilles ne fasse plus de fautes d'orthographe, l'instituteur le pousse à reprendre le mot dès son origine latine, et à suivre sa métamorphose au fil du temps afin que Gilles comprenne l'histoire de la langue et des mots pour réussir l'exercice de la dictée sans faire aucune faute. François Vezin exprime que «le passé ne cesse d'être et par là de venir à

95 Jean-Luc- Seigle, Op.cit. p. 98 
nous, qu'il se prolonge dans le présent agissant en lui et lui donnant figure $»{ }^{96}$

Gilles découvre également que le passé et le présent sont deux réalités dont les événements se répètent, de telle sorte que les dates ne sont selon lui que des nominations : «l'histoire est vertigineuse: les dates, si on y réfléchit bien, ne sont qu'une manière de donner des noms au temps pour ne pas se perdre. ${ }^{97}$

Kant affirme que «le présent n'est pas seulement lié avec tous les éléments du passé, mais mis en action et pour ainsi dire multiplié, pour produire un concept du présent (...). Ainsi le fait de l'expérience passée est-il dans l'observation du temps présent un fondement de la connaissance de l'état présent du monde, (...). $\gg>^{98}$

A la lumière de ce constat, le personnage prévoit le futur grâce aux incidents du passé. Gilles comprend aussitôt que la mission de son frère Henri sera accomplie. La France l'envoie comme soldat dans l'Algérie colonisée pour calmer les émeutes des Algériens contre les Français. En évoquant le passé, Gilles déduit que la France acceptera de se retirer sans combattre pour

96 Martin Heidegger, Op.cit., p. 589

97 Jean-Luc- Seigle, Op.cit. p.97

98 Michel Puech, Kant et la causalité ét étude sur la formation du système critique, Librairie philosophique, 1990, Paris p. 428 
ne pas reproduire l'erreur qui avait causé sa défaite en Indochine en 1947.

En outre, il revient à l'histoire pour relater et interpréter l'expédition pacifique de Christophe Colomb lancée par l'Espagne du Xv $v^{\mathrm{e}}$ siècle à la découverte d'autres territoires outreAtlantique, en la comparant à celle de son frère Henri envoyé en Algérie par la France du $\mathrm{Xx}^{\mathrm{e}}$ siècle. Les Algériens sont alors assimilés aux Indiens de l'ancienne Amérique aux corps peints et emplumés qui ont accueilli Colomb à son arrivée.

Gilles constate d'ailleurs que le passé reflète le présent et affirme que : "Tout était parfaitement clair, cohérent et juste. Cela ne faisait aucun doute, Henri était un des hommes de Christophe Colomb et la France l'Espagne du XVe siècle. Point de cruauté dans tout ça (...), seulement de la découverte, de la justice et du christianisme. ${ }^{99}$ Il creuse ainsi l'immédiateté de l'instant par le passé qui ne prend son sens qu'à partir du présent.

À ce propos, le grand philosophe Edmund Husserl parle d'une intentionnalité à double sens: «les impressions sont déplacées comme suite d'instants présents, ce qui s'accompagne d'un autre déplacement des rétentions qui se rapporte sur chaque impression actuelle et lui permet de vivre le temps

99Jean-Luc- Seigle, Op.cit. p. 87

$-95-$ 
comme une forme de tous les événements ${ }^{100}$. Ainsi, les frontières du temps sont abolies et ne constituent qu'une succession de faits qui se relatent par leur affinité et leur proximité.

Par ailleurs, on interprète les erreurs des militaires en se référant à la littérature française alors que les textes fondateurs de l'Iliade et de l'Odyssée en sont truffés. En remontant quelques années en arrière, Gilles trouve une réponse à cette question contemporaine dans le passé politique de la France.

La défaite contre les Allemands à l'issue de la première guerre mondiale ainsi que la mort de milliers de soldats français durant la seconde guerre mondiale prouvent un manque d'exploits par rapport à l'époque d'Ulysse et d'Achille. Gilles Justifie en disant :

«Toujours la même histoire qui se répète, toujours les mêmes abus, toujours les mêmes incompétences, toujours les mêmes intérêts cachés, et toujours les mêmes à mourir. En 1940, au Nord, l'Armée française est en déroute, non faute de moyens, comme on l'a si souvent dit, ni d'hommes; mais fautes d'ordres qui ne sont pas donnés aux bons moments ou pas donnés du

100 Tatjana Barazon, Op.cit. 
tout $\gg{ }^{101}$ Alors, le personnage de Seigle qualifie cette situation politique comme la honte de l'armée française.

En outre, la Ligne Maginot, imaginée par André Maginot, ministre de la Défense lors de la première guerre mondiale, s'est finalement révélée inefficace, et les Allemands ont envahi la France malgré cette fortification bétonnée.

Ainsi, Gilles comprend grâce à l'histoire de la France la raison pour laquelle le personnage militaire n'est plus un héros constant dans la littérature moderne bien que le texte fondateur qu'est l'Iliade ait été un texte de batailles.

Il découvre que la majorité des textes contemporains renoncent à illustrer les douleurs et les cris des veuves de guerre de la France vaincue près des tombeaux de leurs morts. Les textes littéraires doivent toujours montrer la France victorieuse sans défaites ni défauts.

Dominique Kunz Westerhoff signale que «L'extase de la mémoire involontaire effectue donc une synthèse, elle distille dans l'alambic du souvenir l'essence commune du passé et du présent, qui est aussi une essence du moi Ces instants de révélation mnémonique constituent des «instants purs»qui dépassent ce que le temps a d'inachevé et d'insignifiant, pour

101 Jean-Luc- Seigle, Op.cit. p. 241

-97- 
accéder à une forme d'omnitemporalité, à une complétude du temps ou à un temps absolu. ». ${ }^{102}$

De ce fait, Gilles met en profit ce qu'il a appris du passé alors que les frontières entre passé et présent sont abolies. Il prend comme modèle ses prédécesseurs que l'histoire présente leurs expériences. Il explique à ses étudiants comment exploiter le passé en se rappelant des épreuves des ancêtres. Le héros prend comme exemple André Maginot qui a profité du passé en prenant comme archétype Vauban, ingénieur du XVIII siècle qui a construit soigneusement des fortifications pour protéger la France.

Le grand politicien français assimile la compétence de son confrère de jadis pour sa nouvelle construction de la ligne Maginot. Il se rappelle à ce moment des mots d'Emmanuel Kant, philosophe du XVIII siècle, pour affiner son projet de défense inviolable, qui souligne que : "la colombe a besoin de l'air contre lequel elle résiste de toutes ses forces pour pouvoir voler, sinon elle s'écrase au sol» ${ }^{103}$. Gilles démontre à la nouvelle génération que les grandes personnalités de l'Histoire comme

102 Dominique Kunz Westerhoff, La mémoire en toutes lettres, Université de Lausanne, Suisse, 2007. En (https://www.unil.ch/fra/fr) 103 Jean-Luc- Seigle, Op.cit. p. 237. 
André Maginot profitent des expériences du passé et suivent ce que les grands philosophes ont préconisé.

Ingrid Holtey souligne également que «(...) afin de nous orienter dans le temps historique "nous nous livrons à un pronostic sur l'avenir dont la lumière se reflète sur le présent pour l'éclairer. Ce que nous appelons "passé” n'est qu'une reconstruction qui apparaît derrière le présent dans cet éclairage pragmatique $»^{104}$

De ce fait, la réconciliation avec le temps permet plusieurs rencontres avec d'autres personnalités et renforce la volonté d'apprentissage et d'interprétation de la vie humaine. L'homme devient ainsi libre à manipuler la durée et à rejoindre ses confrères jusqu'au moment où se crée une profonde affinité avec une conception de l'homme soutenue par les empreintes du passé

Alors, Gilles et son confrère, le maître d'école, savent que le temps est inséparable. Il reste continu, accidentel et nouveau, parce qu'il rassemble plusieurs faits pour

faire une synthèse ouvrant sur la connaissance. Or vivre et méditer sur les deux temps reste un mouvement de pensée

104 Ingrid Holtey, "Théorie de l'histoire et représentations du temps contradictoires: à propos d'une étude de Heinz Dieter Kittsteiner », Revue Trivium, no 9, 2011, p. 7 en (https://trivium.revues.org) 
persistant et ininterrompu qui s'efforce de concevoir le monde d'une façon généralisée.

Friedrich Hegel souligne que «L'histoire est le processus par lequel l'esprit se découvre lui-même. On doit chercher à découvrir et identifier la raison dans l'histoire, le passé est le jeu d'une dialectique dont se sert la Raison pour se réaliser, un processus d'autoréalisation de l'Idée, dont la fin est la liberté humaine $\gg .{ }^{105}$

Médiateur entre le passé et le présent, Gilles apprend à ses contemporains à travailler le temps, à mettre le passé au service du présent, à organiser le passé selon les impératifs du quotidien.

Ainsi, vivre en communiquant avec les deux temps rend le personnage de Seigle contemporain d'un temps extrêmement ancien qui lui permet d'entrer dans les siècles et de toucher les morts, alors qu'il s'infiltre dans le présent pour les revivre de manière synchronique.

105 Robert Paris, "Quelle est la philosophie de l'Histoire de Friedrich Hegel ? », Op.cit. No 4740, 2015. 


\section{Conclusion}

Notre étude montre qu'on peut renouer avec le temps sans le défier. Cette combinaison entre l'homme, le passé et le présent empêche tout sentiment d'angoisse et de perte.

Jean-Luc Seigle démontre que lorsque la perception du temps est objective, le temps renonce à son statut de destructeur ; en revanche, lorsqu'on l'observe de manière subjective, il nous défie et nous détruit.

L'étude nous révèle que ceux qui vivent le passé choisissent un temps stagnant et immobile, tandis que pour ceux qui vivent le présent, l'instant se précipite de façon excessive et démesurée.

L'auteur nous apprend que si nous profitons de la part positive du temps que sont les épreuves de l'histoire, nous procédons à l'interprétation des faits du quotidien, et nous vivons gaiement. En revanche, l'homme qui souffre est celui qui n'observe que la part négative du temps : sa fuite, son passé souffrant, et son présent fugitif.

L'auteur nous invite à vivre un temps homogène et synchronique où on ne sent ni sa rapidité ni sa stagnation, une vie qui garantit notre liberté en évitant d'être captif d'un temps dominant. 
Pour cela, il faut profiter du passé au lieu de le subir, en donnant plus d'épaisseur à l'instant sans tenir compte de sa fugacité. Ce cycle temporel nous fait sentir que nous vivons un temps infini.

Notre recherche révèle également que le présent n'est que le miroir du passé, et que l'homme doit prouver sa capacité cognitive à gérer la présence des deux. Le temps n'est qu'une répétition de faits, d'attitudes, et l'homme tire profit des épreuves vécues.

Jean-Luc Seigle nous transmet son message, que nous pouvons mettre en pratique : le temps sert l'homme dès lors que ce dernier respecte sa linéarité et l'investit à chaque moment de sa vie. 


\section{Bibliographie}

\section{I) Corpus}

- Seigle Jean-Luc En vieillissant les hommes pleurent, Flammarion, Paris 2012

\section{II) Ouvrages Critiques :}

- Bouton, Christophe. Temps et esprit dans la philosophie de Hegel : de Francfort à Iéna, Vrin, Paris, 1991

- Comte-Sponville, André. L'Entre-Temps, Puf, Paris,1999

- Derrida, Jacques . La Voix et le Phénomène, PUF, Paris, 1967.

- Dadoun, Roger. La violence, essai sur l'homo violens, Hatier, Paris, 1993

- Gocel, Véronique. Histoire de Claude Simon : écriture et vision du monde, Peeters, Paris, 1996

- Heidegger, Martin. Etre et Temps, par François Vezin, Gallimard, Paris, 1990

- Heidegger, Martin. (trad. François Vezin), Etre et Temps, Paris, Gallimard, 1986

- Hume, David. Traité de la nature humaine, livre I et appendice, traduction de Philippe Baranger et Philippe Saltel, Flammarion, Paris, 1995. 
- Kristeva, Julia. Navarro, Marie-Christine. Au risque de la pensée, L'Aube, 2001

- Malraux, André. Lazare. Le Miroir des Limbes. Gallimard, France, 1974.

- Puech, Michel. Kant et la causalité $\square$ : étude sur la formation du système critique, Librairie philosophique, Paris, 1990.

- Proust, Marcel .A la recherche du temps perdu, Gallimard, Paris, 1919

- Tomasella, Saverio. Le sentiment d'abandon, Eyrolles, Paris, 2010

- T Hall, Edward. La Dance de la Vie, Temps culturel, Temps Vécu, Seuil, Paris, 1992

- Vezin, François. Martin Heidegger, Etre Et Temps, Paris, Gallimard,1986

- Weyembergh, Maurice. Albert Camus, ou, La mémoire des origines, De Boeck université, le point philosophique, 1997.

- Weyembergh, Maurice. Albert Camus, ou, La mémoire des origines, De Boeck université, Paris, 1998,

- Webiographie : Articles consultés sur Net: 
- Albert, Henri. Frédéric Nietzsche. Considérations inactuelles. T. 1 Mercure de France, Paris, 1873 en (http://gallica.bnf.)

- Barazon, Tatjana. L'expérience du temps De la phénoménologie à Bergson, Esprit d'avant, Chronique, No 2, 2008 en (http://www.espritdavant.com)

- Buchon, Jean Alexandre. Choix d'ouvrages mystiques, dans «Confessions de St -Augustin»- Livre XI, A. Desrez, 1840 en (https://books.google.com.eg)

- Cazenave, Michel. «Le temps de la physique », Etienne Klein, Dictionnaire de l'ignorance, Albin Michel, France, 1998, en Centre international de recherches et études transdisciplinaires (CIRET).no 12, 1998 (http://cirettransdisciplinarity.org)

- Charlet, Emilie L'Objet, la Relation., revue Agôn, dossiers no 4, 2011 en (http//agon.ens-lyon.fr)

- Gori, Roland. La mémoire freudienne : se rappeler sans se souvenir, en Cliniques méditerranéennes, Eres, $n^{\circ}$ 67, 2003. ( https://www.cairn.info)

- Gruyelle, Emilie. Psychologie, « Je Garde tout », 2008, p.1 en (http://www.psychologies.com)

- HEGEL, Friedrich. Phénoménologie de l'esprit, Tome I, Traduction de Jean Hyppolite, Montaigne, Paris,1807en (https://books.google.com.eg)

- Haar Michel. Martin Heidegger, Le concept de temps, 1924, dans Cahiers de l'Herne, $\quad \mathrm{N}^{\circ}$ 45,1983, En (https://fr.wikipedia.org)

- Holtey, Ingrid. «Théorie de l'histoire et représentations du temps contradictoires : à propos d'une étude de Heinz 
Dieter Kittsteiner », en Revue Trivium, no 9, 2011 (https://trivium.revues.org)

- Jacob, Christian. Retour vers le Futur, «Rassembler la mémoire », en Revue Diogène, no 196, 2001. (https://www.cairn.info)

- Klein, Etienne. Le Temps de la Physique, Centre International de recherche transdisciplinaire(CIRET), no 12, 1998 en (http://ciret-transdisciplinarity.org)

- Manon, Simon. Visage de la folie Humaine, Nous ne tenons plus au présent Pascal, en Philolog, Cours de philosophie, 2009. En (http://www.philolog.fr)

- Nguyen, Thi Tu Huy. La néantisation du temps chez Robbe-Grillet, Revue Revel unice , Université Nice, no 22, 2008. (URL : http://revel.unice.fr/loxias)

- Paris, Robert Matière et révolution, La mémoire, une reconstruction du passé tournée vers le futur, no 497, 2008, en (http://www.matierevolution.fr)

- Paris, Robert. «Quelle est la philosophie de l'Histoire de Friedrich Hegel ? », en Matière et révolution, no 4740, 2015. (www.matierevolution.org)

- R. Kelly, Michael. La phénoménologie aristotélicienne du Temps : quand l'Esprit «dit» les «maintenants », en Methodos, Savoirs et Textes, no 9, 2009. (https://methodos.revues.org)

- Schärer, Martin R. La relation homme-objet exposé, Revue Publics et Musées, No 15,1999. (www.persee.fr)

- Schaïr, Martin R, La relation homme- objet exposée : théorie et pratique d'une expérience muséologique, Revue 
Publics et Musée, 1999, volume 15, numéro 1, en (http://www.persee.fr)

- Schürch, Franz- Emmanuel. L'Etre, l'étant, le Néant, Revue de Métaphysique et de Morale, presses universitaires de France, no3, 2008 (https://www.cairn.info)

- Vetö, Miklos. L'eidétique de l'espace chez MerleauPonty, revue Archive de philosophie, Université de Poitiers, article no3 , 2008, en (https://www.cairn.info)

- Westerhoff, Dominique Kunz . La mémoire en toutes lettres, Université de Lausanne, Suisse, 2007.( https://www.unil.ch) 
-108 -

PDF created with pdfFactory Pro trial version www.pdffactory.com 